\title{
Star clusters in M 33
}

\section{A new survey from deep HST images ${ }^{\star}$}

\author{
R. Chandar ${ }^{1}$, L. Bianchi ${ }^{1,2}$, and H. C. Ford ${ }^{1}$ \\ 1 Department of Physics \& Astronomy, The Johns Hopkins University, 3701 San Martin Dr., Baltimore, \\ MD 21218, USA \\ e-mail: rupali@pha.jhu.edu; bianchi@pha.jhu.edu; ford@pha.jhu.edu \\ 2 Astronomical Observatory of Torino, 10025 Pino Torinese, TO, Italy
}

Received 2 June 2000 / Accepted 14 November 2000

\begin{abstract}
We have detected 102 star clusters in M 33, from 35 deep Hubble Space Telescope (HST) WFPC2 fields taken from our program and from the HST archive. Twenty-eight fields have $V$ and $I$ band imaging, and an additional seven fields are imaged in a single $V$ filter. Eighty-two of the clusters were previously unknown. Integrated photometry reveals that 25 clusters have $(V-I)$ colors typical of those found in Galactic globular clusters (only ten of these objects were previously known). Our discovery of 15 new globular cluster candidates increases previous estimates of the old cluster population in M 33 by $\sim 60 \%$. An additional eleven objects (only two were previously known) have colors expected for intermediate age clusters. The new clusters have a range of ages from 6 million years to $>15$ Gyrs, and masses between $10^{2} M_{\odot}-10^{6} M_{\odot}$, although these parameters are estimated from only one color. The number of new clusters detected more than doubles our previous sample (Papers I and II), and extends coverage to older objects and new off-spiral arm locations in M 33. The luminosity function for old M 33 clusters shows a peak at $M_{V} \sim-7.0$, nearly half a magnitude fainter than found in the Galactic and M 31 globular cluster populations. The luminosity function for intermediate age objects shows no turnover down to $M_{V} \sim-6.6$. We estimate the total number of globular clusters in M 33 to be $75 \pm 14$. This gives a specific frequency, $S_{\mathrm{N}}$, of $2.07 \pm 0.39$ - significantly higher than found for other late-type spiral galaxies, but in the range found for ellipticals. *
\end{abstract}

Key words. galaxies: individual (M 33) - galaxies: star clusters - galaxies: evolution

\section{Introduction}

At a distance of $\sim 840 \mathrm{kpc}$ (distance modulus $=24.64$; Freedman et al. 1991), M 33 is the only nearby latetype spiral galaxy and, as such, it provides an important link between the cluster populations of earlier-type spirals (e.g., Milky Way and M 31) and the numerous, nearby later-type dwarf galaxies. Ground based work has yielded a limited database of star clusters in M 33 (Christian \& Schommer 1982, 1988; Hiltner 1960; Kron \& Mayall 1960; Melnick \& D'Odorico 1978; Mochejska et al. 1998). Christian \& Schommer $(1982,1988)$ catalogued non-stellar sources by visually examining $\sim 6.7^{\prime \prime} \mathrm{mm}^{-1} \mathrm{di}-$ rect plates of $\mathrm{M} 33$, and obtained ground based $B, V$ and $I$ (or $R$ ) photometry for $\sim 106$ star cluster candidates. Because of crowding in the central regions and bright

Send offprint requests to: $\mathrm{R}$. Chandar

* Table 2 is only available at CDS via anonymous ftp to cdsarc.u-strasbg.fr (130.79.128.5) or via

http://cdsweb.u-strasbg.fr/cgi-bin/qcat?J/A+A/366/498 spiral arm patches, they searched an area $10^{\prime}-18^{\prime}$ from the center of the galaxy. They estimated that M 33 contains only 20-25 "true" globular clusters (GC), based on the results of their survey. Mochejska et al. (1998) detected 35 new clusters in M 33; five of these have $(B-V)_{0} \geq 0.6$ or $(V-I)_{0}>0.78$, colors typical of Galactic GCs.

In previous work we analysed multiband $(U, B$, and $V)$ HST WFPC2 images of $20 \mathrm{M} 33$ fields, covering a range of environments, and including several spiral arm portions and star forming regions. These observations allowed us to resolve structure in even the most crowded spiral arm regions, leading to the detection of 60 star clusters, 49 of which were previously unknown (Chandar et al. 1999a; hereafter Paper I). Clusters at the distance of M 33 are easily distinguished from stellar sources in HST WFPC2 images, as discussed in Paper I. Because three filter observations were available, photometry for stars in the WFPC2 images was used to estimate local reddening (Chandar et al. 1999b; hereafter Paper II). For the 60 clusters in our previous sample, we measured 
integrated magnitudes and colors. Dereddened colors were compared to stellar evolutionary models to estimate age and metallicity for the cluster sample. We found that M 33 clusters formed continuously with ages between 4 million years and 15 Gyrs. Clusters have masses in the range from several $\times 10^{2} M_{\odot}$ to several $\times 10^{5} M_{\odot}$, with a marked trend with age, such that the most massive clusters are missing from the young population. Only seven clusters from our previous sample have integrated $(B-V)_{0} \geq 0.6$, consistent with GC colors. Additionally, 15 young clusters $\left(\leq 10^{8}\right.$ years) have emission detectable in the WFPC2 F170W filter $(1700 \AA)$. The far ultraviolet flux combined with optical magnitudes was used to further constrain reddening and age for young M 33 clusters (Bianchi et al. 1999; Chandar et al. 1999c; hereafter Paper III). King profiles were fit to the brightest cluster radial profiles, and core radii are estimated in the range from $<0.2$ parsecs to $\sim 2$ parsecs, with the few old clusters having larger core radii on average than the young and intermediate populations (Paper II).

By integrating the number density of clusters as a function of radius over the surface of the galaxy, we estimated that there are $\sim 700$ clusters in M 33 (Paper I). However, currently, less than 200 clusters or cluster candidates are known in this galaxy, and most of these have ages $<1 \mathrm{Gyr}$.

Finding additional GC candidates in M 33 is important in order to constrain the early formation and evolution of this galaxy. M 33 is known to have a kinematic halo of clusters (Schommer et al. 1991; Chandar et al. 2001); however, there is some evidence that the halo clusters in M 33 have an age spread which is $2-3$ times larger (10-15 Gyrs) than found in the Milky Way (Sarajedini et al. 1998). This has significant implications for timescales of galaxy formation.

In order to extend the search for clusters in M 33, and particularly to focus on detection of older objects, we searched the HST Archive and found $28 \mathrm{M} 33$ fields imaged with WFPC2 in $V$ (either F606W or F555W), and $I(\mathrm{~F} 814 \mathrm{~W})$ bands. An additional seven fields have long exposures (at least $300 \mathrm{~s}$ ) in a single $V$ (F555W or F606W) filter. The red filter set is particularly useful for detecting and studying older objects. Furthermore, the observations used in this study are deeper than used in Papers I and II, ideal for detecting faded, old clusters. For example, the 10 fields imaged for GO5914 (see Table 1) have two $1300 \mathrm{~s}$ exposures in the F555W and F814W filters. All fields were visually searched for populous clusters, resulting in the detection of 102 objects.

This paper is organized as follows: Sect. 2 describes the data and photometric reduction and measurements for our catalog, and Sect. 3 discusses the luminosity function for old and intermediate age clusters, the color magnitude diagram, cluster age and mass distributions, and specific frequency of globular clusters in M 33. The results are summarized in Sect. 4.

\section{The data: Reduction, cluster detection, and photometry}

\subsection{The observations}

Table 1 contains general data properties, such as the field identification, Proposal ID, the number and length of exposures in the various filters, and the number of clusters found in each field. The location in M 33 of the HST WFPC2 fields is shown in Fig. 1. Surveyed fields in this study cover a larger percentage of off-spiral arm and outer galaxy regions (out to $6 \mathrm{kpc}$ ) than our previous work (Paper I). These locations are well suited to the search for new globular cluster candidates, since faint red clusters are more difficult to detect when projected against the bright, variable background from spiral arms. All images were calibrated through the WFPC2 pipeline at STScI. These reduction steps include: bad pixel masking, A/D correction, bias and dark subtraction, and flat field correction. We checked the alignment of different filters and exposures in each field, and combined multiple exposures in order to eliminate cosmic rays and increase signal to noise. The IRAF task COSMICRAYS was run on single exposure images to eliminate high signal cosmic rays.

The HST WFPC2 resolution (pixel sizes are $0.1^{\prime \prime}$ in the three Wide Field CCD's and 0.0455" in the Planetary Camera CCD, where $1^{\prime \prime}$ corresponds to 4.1 pc in M 33) makes it easy to distinguish individual stars from clusters at the distance of $\mathrm{M} 33$. The images were visually searched for clusters in available filters. All clusters have radial profiles which are extended when compared with a stellar profile (details of the separation of clusters and stars are as given in Paper I). The entire cata$\log$ (Table 2) from this work is only available in electronic form. Columns 1-4 contain the object number, RA, DEC, and projected galactocentric distance (in $\mathrm{kpc}$ ). Column 8 contains morphological comments and cross identifications with other works. We begin numbering clusters at 61 , since this catalog is a continuation of the previous survey (Paper I). Figure 2 shows $V$ and $I$ filter images $\left(12^{\prime \prime}\right.$ per side) of four new GC candidates in our catalog.

\subsection{Photometry}

In order to understand the nature of clusters and determine important parameters such as total luminosity and integrated color, an aperture size must be chosen. We used a $2.2^{\prime \prime}$ radius aperture in order to measure the total $V$ magnitude, since we found previously that this was the best compromise between the optimum signal to noise ratio and the asymptotic growth of the computed magnitudes for M 33 clusters in HST WFPC2 images (Paper I). While this aperture size appeared appropriate for most clusters in our new catalog, there are several objects which were obviously more extended. For these clusters, the aperture size used to measure the total $V$ magnitude is noted in Table 2 . The radius used to measure integrated 
Table 1. Summary of HST WFPC2 fields used in this survey

\begin{tabular}{ccccccc}
\hline$\#$ & FIELD & PropID & \multicolumn{2}{c}{ Filters Exposure Time $[\mathrm{s}]$} & Number \\
& & & F555W $(V)$ & F606W $(V)$ & F814W $(I)$ & of clusters \\
\hline \hline 1 & M 33-AS1 & 5914 & $2 \times 1300$ & $\ldots$ & $2 \times 1300$ & 9 \\
2 & M 33-AS2 & 5914 & $2 \times 1300$ & $\ldots$ & $2 \times 1300$ & 11 \\
3 & M 33-AS3 & 5914 & $2 \times 1300$ & $\ldots$ & $2 \times 1300$ & 4 \\
4 & M 33-AS4 & 5914 & $2 \times 1300$ & $\ldots$ & $2 \times 1300$ & 5 \\
5 & M 33-AS5 & 5914 & $2 \times 1300$ & $\ldots$ & $2 \times 1300$ & 8 \\
6 & M 33-AS6 & 5914 & $2 \times 1300$ & $\ldots$ & $2 \times 1300$ & 6 \\
7 & M 33-AS7 & 5914 & $2 \times 1300$ & $\ldots$ & $2 \times 1300$ & 2 \\
8 & M 33-AS8 & 5914 & $2 \times 1300$ & $\ldots$ & $2 \times 1300$ & 8 \\
9 & M 33-AS9 & 5914 & $2 \times 1300$ & $\ldots$ & $2 \times 1300$ & 2 \\
10 & M 33-AS10 & 5914 & $2 \times 1300$ & $\ldots$ & $2 \times 1300$ & 7 \\
11 & M 33-JM1 & 5397 & $1 \times 500$ & $\ldots$ & $1 \times 500$ & 4 \\
12 & M 33-JW1 & 5236 & $2 \times 500$ & $\ldots$ & $2 \times 10$ & 1 \\
13 & M 33-KM1 & 6640 & $2 \times 800$ & $\ldots$ & $3 \times 160$ & 1 \\
14 & M 33-KM2 & 6640 & $2 \times 800$ & $\ldots$ & $2 \times 800$ & 2 \\
15 & M 33-KM3 & 6640 & $2 \times 800$ & $\ldots$ & $2 \times 800$ & 1 \\
16 & M 33-KM4 & 6640 & $2 \times 800$ & $\ldots$ & $2 \times 800$ & 4 \\
17 & M 33-KM5 & 6640 & $2 \times 800$ & $\ldots$ & $2 \times 800$ & 2 \\
18 & M 33-KM6 & 6640 & $2 \times 800$ & $\ldots$ & $2 \times 800$ & 1 \\
19 & M 33-RG1 & 5369 & $\ldots$ & $1 \times 1200$ & $1 \times 2100$ & 1 \\
20 & M 33-MR1 & 5464 & $2 \times 400$ & $\ldots$ & $2 \times 400$ & 1 \\
21 & M 33-SC1 & 8059 & $\ldots$ & $1 \times 200$ & $1 \times 160$ & 0 \\
22 & M 33-SC2 & 8059 & $\ldots$ & $1 \times 300$ & $1 \times 300$ & 1 \\
23 & M 33-SC3 & 8059 & $\ldots$ & $1 \times 300$ & $1 \times 300$ & 1 \\
24 & M 33-SC4 & 8059 & $\ldots$ & $1 \times 200$ & $1 \times 300$ & 0 \\
25 & M 33-SC5 & 8059 & $\ldots$ & $1 \times 200$ & $1 \times 300$ & 1 \\
26 & M 33-SC6 & 8059 & $\ldots$ & $1 \times 200$ & $1 \times 300$ & 2 \\
27 & M 33-SC7 & 8059 & $\ldots$ & $1 \times 200$ & $1 \times 400$ & 1 \\
28 & M 33-SC8 & 8059 & $\ldots$ & $1 \times 200$ & $1 \times 300$ & 1 \\
29 & M 33-SC9 & 8090 & $\ldots$ & $1 \times 700$ & $\ldots$ & 4 \\
30 & M 33-SC10 & 8090 & $\ldots$ & $2 \times 1000$ & $\ldots$ & 4 \\
31 & M 33-JH1 & 5179 & $1 \times 350$ & $\ldots$ & $\ldots$ & 2 \\
32 & M 33-LB1 & 5494 & $\ldots$ & $1 \times 350$ & $\ldots$ & 3 \\
33 & M 33-LB2 & 5494 & $\ldots$ & $1 \times 350$ & $\ldots$ & 0 \\
34 & M 33-LB3 & 5494 & $\ldots$ & $1 \times 350$ & $\ldots$ & $\ldots$ \\
35 & M 33-LB4 & 5494 & $\ldots$ & $1 \times 350$ & & $\ldots$ \\
\hline
\end{tabular}

colors was chosen by examining each cluster image individually, and is given in Col. 6 of Table 2. Integrated colors were obtained in the given radii in order to avoid any potentially contaminating sources (see Paper I for a more extensive discussion). Mean local background levels were measured around each cluster in an annulus with inner radius of $3.5^{\prime \prime}$ and outer radius of $5.0^{\prime \prime}$. The uncertainty in local background measurements is included in the magnitude uncertainties. The following steps were used to transform WFPC2 instrumental magnitudes in the F555W (or F606W) and F814W filters to Johnson-Cousins $V$ and $I$ magnitudes: (i) After preprocessing through the STScI pipeline, image alignment, cosmic ray rejection, and aperture photometry was performed using the IRAF $^{2}$ package DAOPHOT; (ii) the instrumental magnitudes were

\footnotetext{
${ }^{2}$ IRAF is distributed by the National Optical Astronomy Observatories, which are operated by the Association of Universities for Research in Astronomy, Inc., under cooperative agreement with the National Science Foundation.
}

corrected for the charge-transfer efficiency (CTE) loss, which depends on object position on each CCD (both $x$ and $y$ ), the background counts, object counts, and date of observations. Using the prescription in Dolphin (2000), typical CTE corrections make cluster magnitudes brighter by $\sim 0.003$ to 0.05 magnitudes in our sample. The cluster colors were unaffected by CTE corrections, within the photometric uncertainties. (iii) the corrected instrumental magnitudes were converted to standard JohnsonCousins $V$ and $I$ magnitudes. Using Eq. (8) and Table 7 of Holtzman et al. (1995), the magnitudes were derived iteratively using WFPC2 observations in two filters, with calibrations for the F606W filter substituted from Dolphin (2000). We did not make corrections to the photometry for geometrical distortions in the WFPC2 CCDs, which can affect the total cluster magnitudes at the $\sim 2-3 \%$ level, but will have negligible effect on cluster colors and hence age estimates. If only one filter (F555W or F606W) is available, then the zeropoint given in Dolphin (2000) was used. 


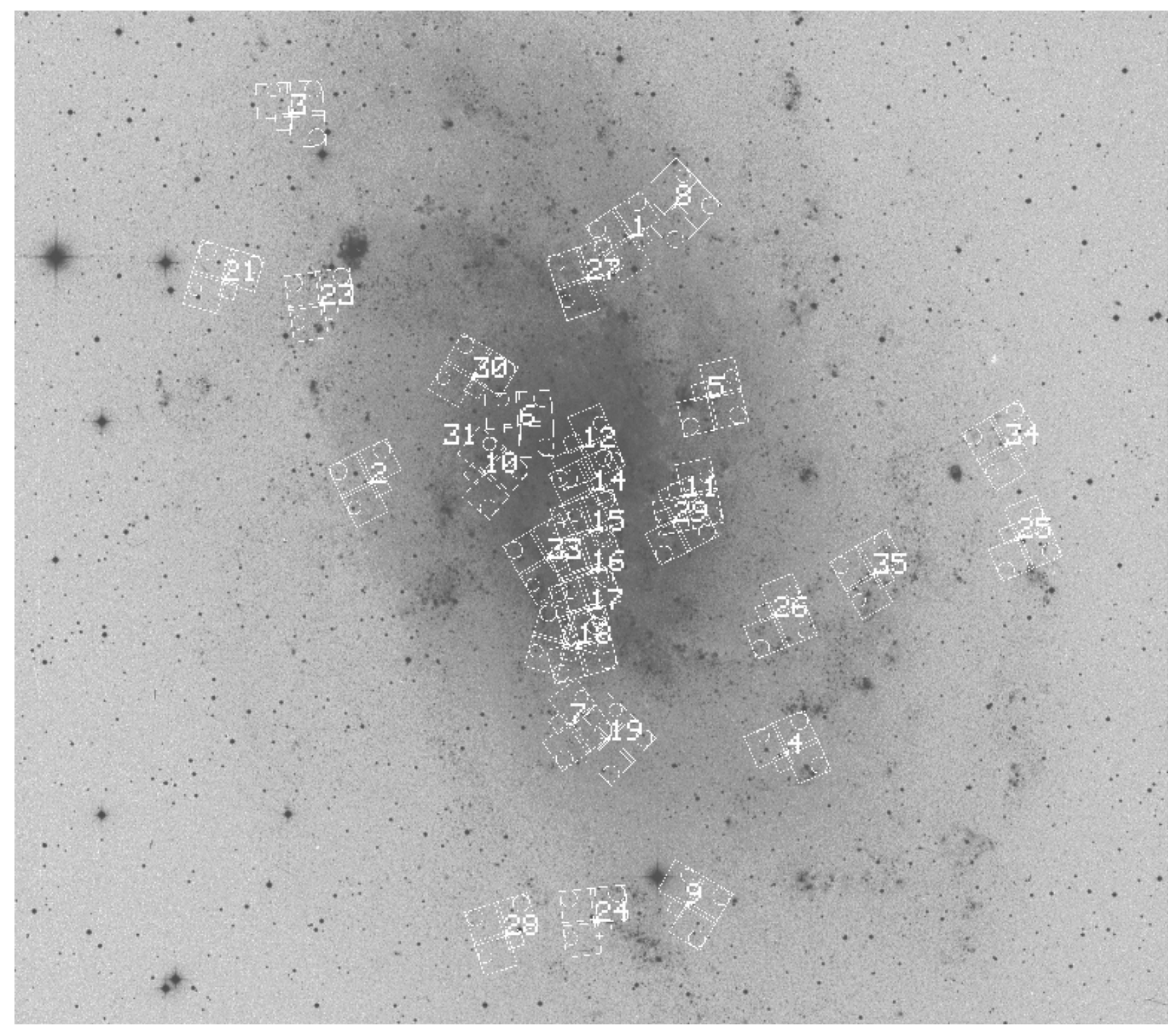

Fig. 1. $45^{\prime} \times 45^{\prime}$ Digitized Sky Survey image of M 33 with the HST fields used in this study superposed and labeled. Fields 12 , 13, and 20 overlap substantially, so in order to avoid confusion only field 12 is shown and labeled in this figure. Fields 10 and 22 overlap substantially, so only field 10 is shown, and fields 18 and 32 overlap, so only 18 is marked

Integrated photometric properties of the clusters are compiled in Cols. 5-7 of Table 2. No reddening correction has been applied to the photometry in this table. The uncertainties given for the $V$ magnitude are obtained from IRAF's PHOT task. These values take into account the object count rate, sky variance, and instrument gain. Uncertainty in color (in the given color radius) is estimated by propagating uncertainties in $V$ and $I$, i.e. $\left.\sigma_{(V-I)}=\left[\left(\sigma_{V}\right)^{2}+\left(\sigma_{I}\right)^{2}\right)\right]^{1 / 2}$, to reflect the error in the two filter measurements.

The use of a fixed aperture to measure cluster magnitudes does not convey any information on relative cluster sizes. Because structural parameters such as core radius are an important diagnostic of the conditions present at cluster and galaxy formation, we give in Col. 11 of Table 2 approximate core radii $\left(r_{\mathrm{C}}\right)$ in parsecs, for the clusters in our current sample. Surface brightness profiles were constructed for each cluster from $V$ band images, and the $F W H M$ measured. This $F W H M$ was then converted to $r_{\mathrm{C}}$ according to the equation $r_{\mathrm{C}}=-0.021+0.50 * F W H M$, from CBF99b. The conversion from measured $F W H M$ to core radius for M 33 clusters was determined by creating a number of synthetic clusters by convolving single mass King profiles (of varying structural parameters) with WFPC2 PSFs. The FWHM was measured for these synthetic images to calibrate the measured $F W H M$ with intrinsic core radius, as described in CBF99b.

Because the data sample regions of M 33 at different brightnesses, and have different exposure times and filters, it is not possible to rigorously set a completeness limit applicable to the entire catalog presented here. Experiments show that we can easily detect typical compact clusters 

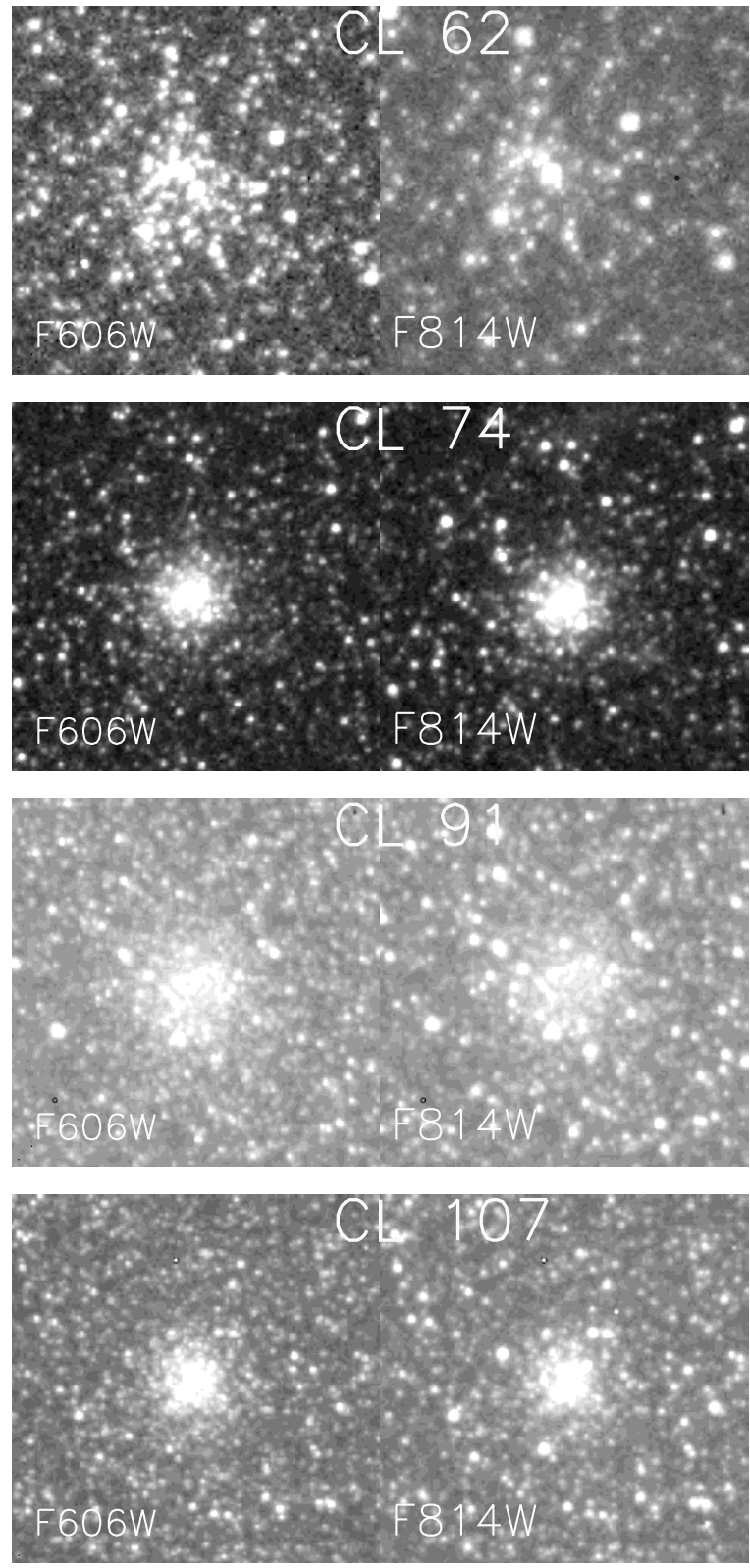

Fig. 2. $12^{\prime \prime} \times 12^{\prime \prime}$ finding charts are shown for four newly discovered globular cluster candidates

down to $m_{V} \sim 19.7$ in the first ten fields, which are the deepest. In these fields, we can usually find clusters to $m_{V} \sim 20.0$. Additionally, fields imaging the outer portions of M 33 have lower background levels, and clusters can be detected to at least $m_{V} \sim 19.7$. By comparison, the typical detection limit of fields in our previous work (Papers I and II) is $m_{V} \sim 19.0$.

Twenty clusters in our survey have been previously detected by Christian \& Schommer (1988) or Mochejska et al. (1998). Columns 9 and 10 of Table 2 give the photometric comparison for overlapping objects, and Fig. 3 shows measurements to be in relatively good agreement for these objects. There is a small zeropoint offset $(\Delta V=$ 0.01 magnitudes) between this work and ground based photometry. We measure slightly fainter magnitudes due to the smaller aperture used in our work. All but four of the objects differ by less than 0.1 magnitudes in $V$, and there is no trend of larger discrepancy with fainter magnitudes. The HST WFPC2 images of clusters 106, 114, and 150 all show crowding, with obvious non-cluster sources outside our aperture, but within that used by Christian \& Schommer (1988), resulting in a larger difference in magnitudes. For cluster 152 we measure a brighter luminosity than Christian \& Schommer - the source of this discrepancy is not clear. Color measurements between the works are also in good agreement, with the exception of object 141 , for which we find $(V-I)=0.47$, but Mochejska et al. (1998) find $(V-I)=0.20$.

In order to estimate cluster ages, integrated colors must be corrected for reddening. The fields used in this survey were correlated with those in the Massey et al. (1995) study, which used $U B V$ photometry and optical spectroscopy to derive the local extinction in several M 33 OB associations. None of our fields overlap with regions of extinction higher than $E_{(B-V)} \sim 0.1$. Although many fields are in portions of M 33 not studied by Massey et al. (1995), these are mostly not spiral arms, and thus not likely to have high internal extinction. For all clusters in this work, we assume a reddening value of $E_{(B-V)}=0.1$. Implications of this assumption will be discussed later. The extinction at the central wavelength of the WFPC2 filters, using the optical extinction law of Savage (1979) gives $E_{(\mathrm{F} 555 \mathrm{~W}-\mathrm{F} 814 \mathrm{~W})}=1.5 \times E_{(B-V)}$. Due to the similarities of the central wavelengths of the F555W and F606W filters, the extinction ratio is approximately the same for $E_{(\mathrm{F} 606 \mathrm{~W}-\mathrm{F} 814 \mathrm{~W})}$.

\section{Results}

\subsection{Luminosity function and color magnitude diagram}

Because we have detected a large number of new clusters, many of which are of intermediate age or old, we show in Fig. 4 new luminosity functions for all known (Paper II, Christian \& Schommer 1988; Mochejska et al. 1998; this survey) intermediate and old clusters in M 33. Based on integrated colors of Galactic and Magellanic Cloud clusters and integrated model colors (Bertelli et al. 1994), we divided our sample into age groups. Old objects are defined as having dereddened colors $(B-V)_{0} \geq 0.6$, or $(V-I)_{0}>0.78$, corresponding to ages $>10 \mathrm{Gyr}$. Intermediate age clusters are defined as having dereddened colors $0.4<(B-V)_{0}<0.6$ or $0.70 \leq(V-I)_{0} \leq 0.78$, corresponding to ages between 3 and 9 Gyr. We note that the old M 33 cluster luminosity function is log normal in form, with an apparent peak around $M_{V} \sim-7.0$. However, this value is an upper limit, since with a fainter completeness limit the turnover may move to fainter magnitudes. Because of the inhomogeneous set of observations in which clusters have been detected, we do not attempt to determine the value of the peak in the luminosity function. This would require an estimate of completeness as a function of magnitude, which is also complicated by the 


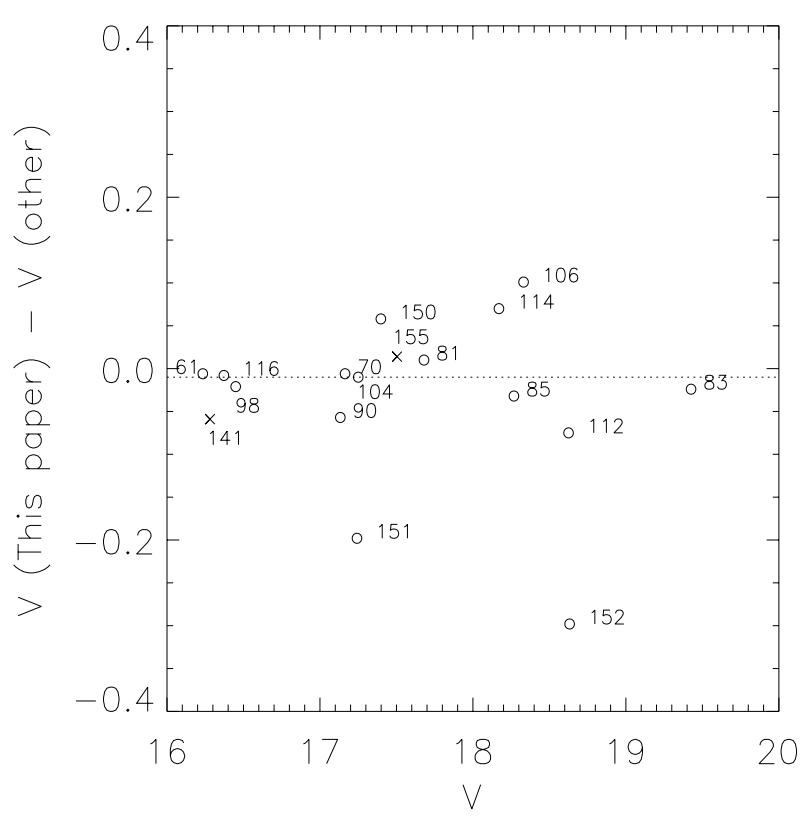

Fig. 3. Comparison between our measured $V$ magnitudes and those of Christian \& Schommer (1988) shown as o's and Mochejska et al. (1998) shown as X's. Photometric measurements differ by more than 0.1 magnitude only for clusters 106 , 114,150 , and 152 . Clusters 106,114 , and 152 are fainter in our survey due to the smaller aperture used for photometry, and crowding seen in HST WFPC2 images near these clusters. However for cluster 152 we measure a brighter $V$ magnitude, and this cluster does not show evidence of crowding in our HST images. The source of discrepancy in the magnitudes is not clear for this object. Excluding cluster 152, we find a mean offset in $V$ magnitudes between our work and ground based surveys of $(\Delta V)=0.01$ magnitude (dashed line), and a median offset of 0.008 magnitudes. This offset in the photometry is due to the smaller apertures used in our work when compared with ground based surveys

spiral structure in M 33. The turnover in the old cluster population is at least several tenths of a magnitude fainter than the value of $M_{V} \sim-7.4 \pm 0.2$ found for the Galactic and M 31 GC systems (e.g., Ashman \& Zepf 1998 and references therein). One possible explanation for the fainter luminosity function of "old" M 33 clusters is that these objects are both several Gyrs younger than their Galactic counterparts, and also more metal rich. From resolved color magnitude diagrams of ten GC candidates in M 33, Sarajedini et al. (1998) find that all but two of the clusters display extremely red horizontal branches. Two clusters form a "second parameter pair", in that they have similar metallicities but very different horizontal branch morphologies. If age is the second parameter (e.g., Sarajedini et al. 1997), than a significant number of M 33 clusters are younger than typical Galactic GCs. Also, from stellar evolution models, a population of young, metal-rich clusters appear bluer and more luminous than old metalpoor clusters until they reach intermediate ages. At later times, these clusters will become fainter and redder than the old population (e.g., see Fig. 13 in Whitmore et al. 1997). However, there is no significant evidence that our "old" M 33 clusters are redder than Milky Way GCs.

The luminosity function for intermediate age M 33 clusters is also shown in Fig. 4. The "turnover" seen in the intermediate age cluster luminosity function is due to incompleteness. Thus this peak value of $M_{V} \sim-6.6$ represents an upper limit to the turnover for these clusters. If more complete surveys establish a turnover in the intermediate age population, it will represent an intermediate form between the brighter turnover seen in the old M 33 cluster population, and the powerlaw form found for young proto-globular clusters in merging galaxy systems (e.g., Whitmore et al. 1999). This progression in form from powerlaw to log normal (with increasingly more luminous turnover magnitudes) with age is expected if destruction mechanisms are responsible for the evolution of the cluster luminosity function. Populous clusters in M 33 may provide the first direct evidence that the cluster luminosity function evolves with age rather than being determined at the time of formation.

Figure 5 shows a $(V-I)_{0}$ vs. $M_{V}$ color magnitude diagram for the clusters detected in this survey. Cluster magnitudes and colors have been corrected for extinction. The large number of clusters with $(V-I)>0.78$ are GC candidates. While several of the new GC candidates are faint $\left(m_{V}>19.0\right)$, there are many others which are fairly luminous $\left(m_{V}<18.5\right)$, and were not detected in previous ground based surveys. These appear to either be compact or located in crowded regions of M 33.

The demographics of clusters presented in this work differ from those discussed in Papers I and II. We find a significantly higher fraction of old objects compared with our previous sample, most likely due to a combination of three factors: the location of the fields (a higher percentage are located off-spiral arms and in outer M 33 portions), the significantly deeper imaging, and the use of redder filters. Out of the 87 clusters imaged in both $V$ and $I$ filters, 36 have $(V-I)_{0} \geq 0.70$ (after applying $E_{(V-I)}=0.15$ ), typical of intermediate age and old clusters. Galactic GCs have $(V-I)_{0}$ redder than 0.78 , and we use this as our criterion to classify old clusters in our sample. Twenty five clusters in our list have $(V-I)_{0}>0.78$ after reddening correction. Thus we have discovered 15 new globular cluster candidates in M 33, and nine new intermediate age cluster candidates. This substantially increases the number of known intermediate age and old clusters in M 33, which has significant implications for the formation history of this galaxy.

\subsection{Age estimates}

Here, ages are estimated by comparing measured $(V-I)_{0}$ cluster colors (corrected for $E_{(B-V)}=0.10$ ) with single stellar population models for two different metallicities: $Z=0.004$ and $Z=0.001$ (Bertelli et al. 1994). Two color $(B-V)$ vs. $(U-B)$ diagrams for our previous sample 

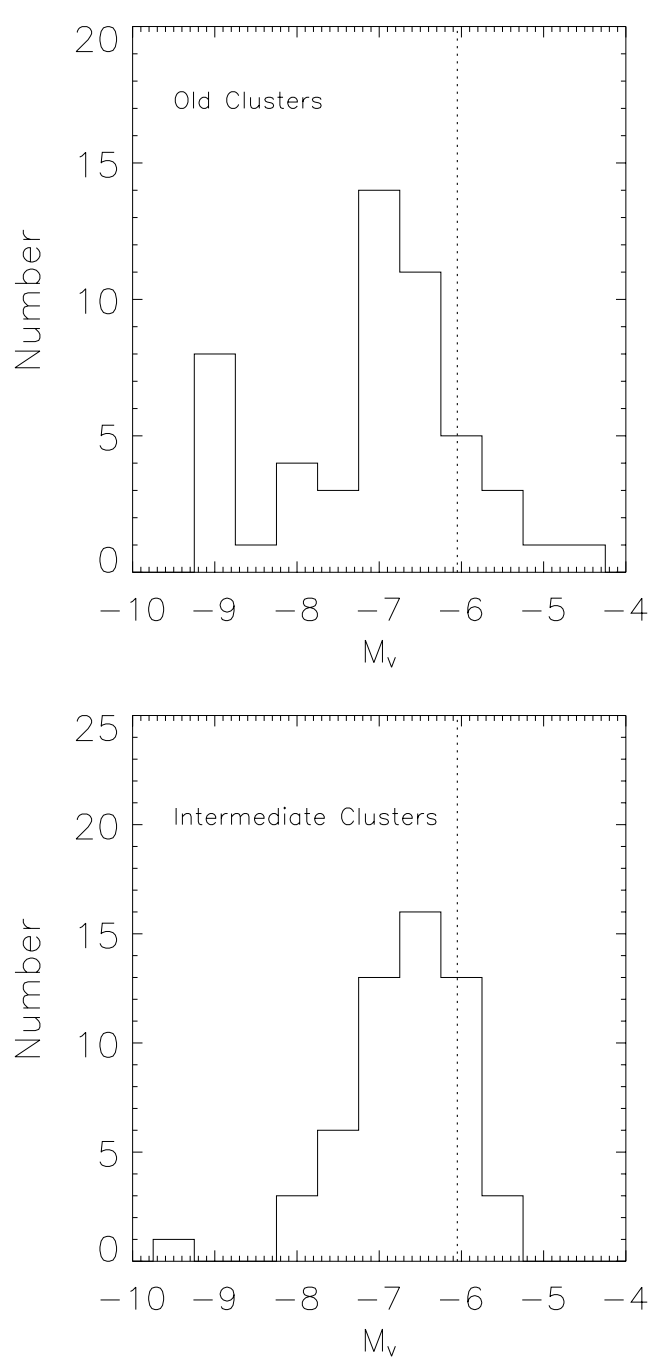

Fig. 4. Luminosity functions for old M 33 clusters (top), defined to have integrated colors $(V-I)_{0}>0.78$ and intermediate age clusters (bottom), defined as $0.70 \leq(V-I)_{0} \leq 0.78$. The dotted lines represent the approximate completeness limits of the combined catalogs (Christian \& Schommer 1988; Mochejska et al. 1998; paper I; this paper) of $m_{V} \sim 19.0$. "Turnovers" are seen at $\sim-7.0$ and $\sim-6.6$ in the old and intermediate age cluster populations, respectively. In the old population this is an upper limit to the turnover magnitude, and the observed turnover in the intermediate age cluster population is due to incompleteness

showed that young M 33 clusters are well represented by the $Z=0.004$ model, and that there is a distinct turnoff to lower metallicity $(Z=0.001)$ in the M 33 cluster population around $10^{9}$ years (Paper II). Therefore we adopt the $Z=0.004$ metallicity model for clusters with dereddened colors $(V-I)_{0}<0.70$, and the $Z=0.001$ metallicity model for clusters with dereddened colors $(V-I)_{0} \geq 0.70$. This color corresponds roughly to an age of $10^{9}$ years according to the Bertelli et al. (1994) models. Due to the relatively large uncertainty associated with age estimates from comparison of one color with single stellar population models, we do not give quantitative age estimates for individual clusters, but rather discuss the statistical properties of the sample. Formal photometric uncertainties are small in most cases, and barely affect age estimates. However, with only one color, ages cannot be well constrained from photometry alone, because it is impossible to accurately disentangle the effects of metallicity and reddening from age without additional information. If the reddening is underestimated for a given cluster, its age would be overestimated (due to the apparently redder color). Another problem with having only one color is accounting for age-color degeneracy within a single metallicity model. In the $Z=0.004$ model, an intrinsic $(V-I)$ color of 0.3 to 0.4 magnitudes gives a degeneracy in ages (in log years) between 7.4 and 8.4. However, this range of color indicates that a cluster is younger than $1 \mathrm{Gyr}$, and thus classified as a young cluster. Note that intrinsic $(V-I)_{0} \sim 0.6$ corresponds to different ages in the $Z=0.004$ and $Z=0.001$ models (log age 7.5 years vs. 8.4 years). Although we cannot measure age more precisely than this, again, both age estimates are less than 1 Gyr. If old clusters are more metal rich than $Z=0.001$, then we have slightly overestimated their age. As a further caution in using ages from this work, note that the F606W filter includes $\mathrm{H} \alpha$; $\mathrm{H} \alpha$ emission is seen in very young clusters $(<10 \mathrm{Myr})$, so age estimates for these very young objects are suspect.

The distribution of cluster ages is shown in Fig. 6 .

\subsection{Mass estimates}

Our previous survey was aimed at detecting young objects, and thus had a small number of old clusters. In order to better constrain the mass range of old M 33 clusters, we estimated masses for our new sample. Measured luminosity and estimated age were combined with theoretical $M / L_{V}$ ratios extracted from the Chiosi et al. (1997) models (as described in Paper III), based on metallicity $Z=0.004$ for clusters with $(V-I)_{0}<0.7$ (ages $\leq 1 \mathrm{Gyr}$ ), and metallicity $Z=0.001$ for clusters older than this age. $M / L_{V}$ ratios do not differ significantly with metallicity. Uncertainty in age (from only having one color) translates to the largest contributing factor in mass uncertainty. Unfortunately the uncertainty in age, and thus mass, is large for clusters older than several billion years. Figure 6 plots mass versus age for the clusters in this survey. The dotted line represents the completeness limit $\left(m_{V} \sim 19.0\right)$ from our previous survey (Paper I), and the dashed line represents the approximate completeness limit of our current sample $\left(m_{V} \sim 19.7\right)$. Here, we have a much larger number of old clusters than in our previous work, and our survey goes deeper by almost 1 magnitude. We find that clusters older than several billion years have a mass range from $10^{4} M_{\odot}-10^{6} M_{\odot}$, similar to that found in the Milky Way. We confirm that the most massive $\left(>10^{5} M_{\odot}\right)$ objects are missing in the cluster population younger than 1 Gyr, as found in Paper II. 


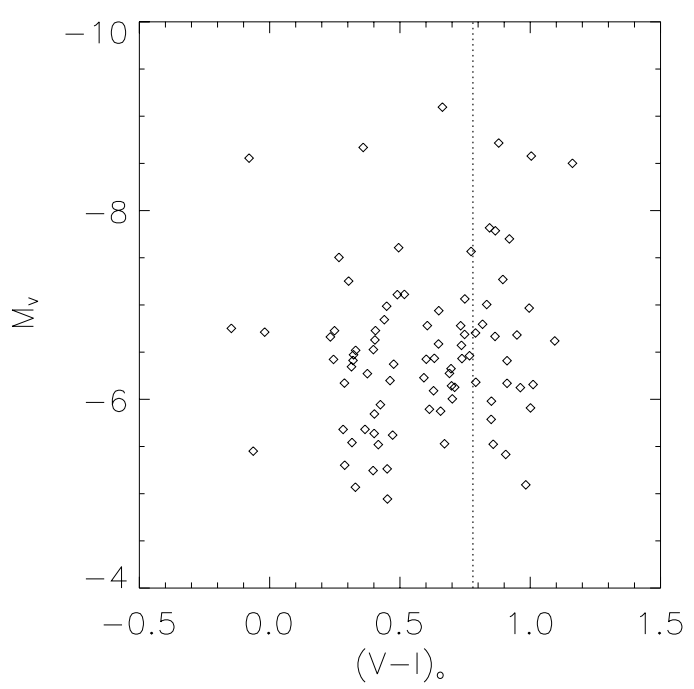

Fig. 5. Dereddened $(V-I)_{0}$ versus $M_{V}$ color magnitude diagram for the current sample of M 33 clusters. The dotted line at 0.78 is the bluest intrinsic $(V-I)$ color for Galactic globular clusters

\subsection{Total number of globular clusters}

Because large portions of M 33 have now been surveyed with HST WFPC2 multiband imaging, we refine the estimate of the total number of GCs expected in this galaxy. We have discovered 15 new clusters in this work which have magnitudes and colors typical of GCs. Combined with candidates from the literature (Paper II, Christian \& Schommer 1988; Mochejska et al. 1998), there are 51 known GC candidates in M 33. We emphasize that all 51 GC candidates have been selected on the basis of integrated colors, such that $(B-V)_{0} \geq 0.6$ or $(V-I)_{0}>0.78$ (candidates which have both $(B-V)$ and $(V-I)$ measurements satisfy the criteria in both colors). However, integrated spectra (with good $\mathrm{S} / \mathrm{N}$ ) of a few old candidates do appear similar to Galactic GCs, with narrow Balmer lines (equivalent widths of $\sim 3 \AA$ ), and unlike spectra of intermediate age Magellanic Cloud clusters (Chandar et al. 2001). Future spectra planned for a large number of the GC candidates will confirm or reject age estimates $\geq 10$ Gyrs for these objects. For now, we assume that these are indeed GCs, and estimate how many such clusters are expected in M 33.

Because M 33 has an inclination of $54^{\circ}-57^{\circ}$, presenting nearly face-on, detecting globular clusters silhouetted against the bright and variable disk is difficult, and makes estimates of incompleteness radially dependent. Because the luminosity function for these clusters differs from the "universal" form found for GC systems in most other galaxies, we cannot correct for incompleteness based on a Gaussian distribution. HST WFPC2 imaging has now covered significant portions of M 33, primarily within $\sim 15^{\prime}$ (see Fig. 1 in this work and in Paper I). One way to estimate the total number of GCs is to correct for the incompleteness in the outer portions of M 33, based on our more complete survey of the inner regions. In Fig. 7 we plot

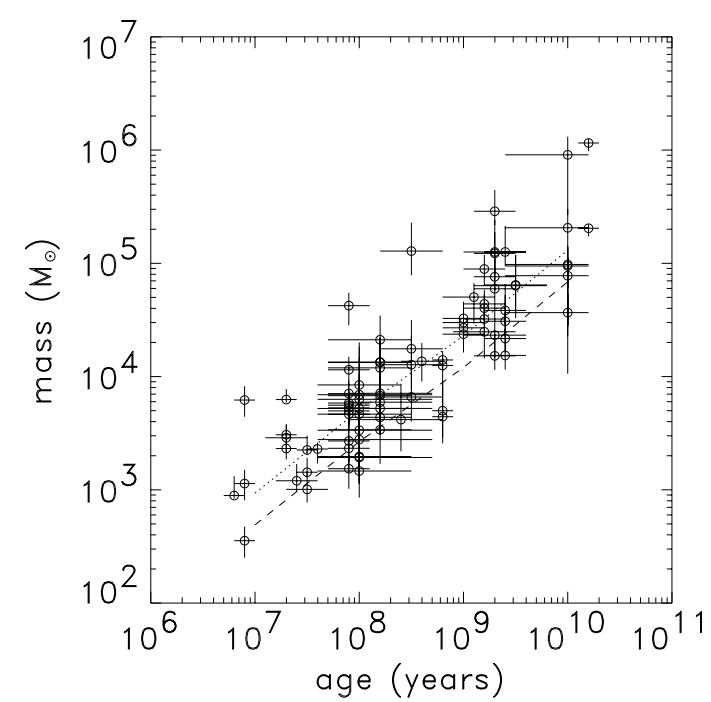

Fig. 6. Mass versus age for the M 33 cluster sample in this paper. The dotted line represents the completeness limit of $m_{V}=19.0$ from our previous survey (Paper II), and the dashed line represents the approximate completeness limit of this work, $m_{V} \sim 19.7$. The deeper imaging in this survey allows us to extend our study to the less massive old cluster population in M 33

the surface density of all known GC candidates. Although technically lower limits, we believe the four inner points are fairly representative of the actual number of GC candidates in this area. In log-log space, the four inner points essentially follow a straight line of slope -1.05 , while the outer 2 points deviate significantly from (fall much below) this line, due to incomplete coverage. We estimate the total number of GCs in M 33 to be 75 by extending this surface density to the outer points. An upper limit to the number of GCs comes from extending the fit out to $35^{\prime}$ $(\sim 8.7 \mathrm{kpc})$.

By using the number of clusters in the inner portions of M 33 to infer the number in the outer portion, we assume that there is no systematic change in the cluster birth or destruction rate with radius. While we cannot make any statement concerning the radial dependence of GC formation, in general, observations suggest that GC survival is enhanced in outer cluster regions. For example, diffuse GCs are only found in the outer regions of the Milky Way, because such clusters, if they formed, would be very easily disrupted in the inner Milky Way. If the survival probability is enhanced for outer old M 33 clusters compared with inner clusters, then our estimate actually represents a lower limit to the total number of GCs expected in M 33.

The surface density slope of -1.05 is similar to that found in some elliptical galaxies, but significantly shallower than the $\sim-2$ found in the Milky Way and M 31 GC systems. The slope of GC surface densities usually flattens out at small radii (e.g., Ashman \& Zepf 1998). If this is the case in M 33, and we simply fit the third and fourth point from the center, we find a slope of -1.95 , very similar to that found for other spiral galaxies. Correcting 


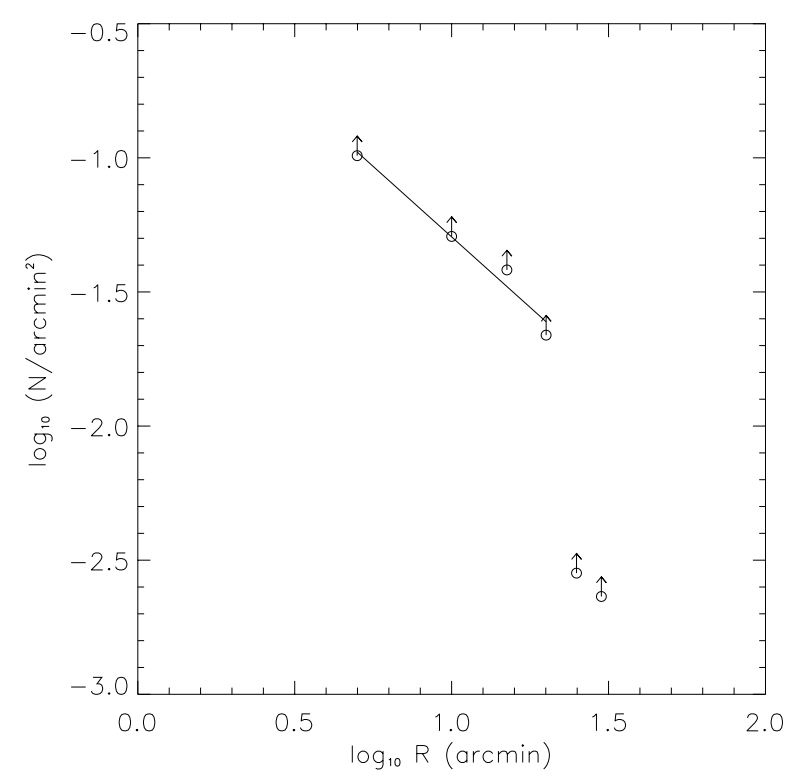

Fig. 7. Surface density distribution of the 51 known globular cluster candidates in M 33. The arrows indicate that these values are lower limits, since there may still be faint, old clusters which have not yet been discovered. The solid line is the best linear fit to the four inner points. The two outer points suffer from the most incompleteness, due to the smaller fractional coverage using deep HST WFPC2 imaging

the incompleteness of M 33 clusters based on this density profile, we would expect $\sim 65$ GCs in M 33. Thus we estimate that there are a total of $75 \pm 14$ GC candidates in M 33.

\subsection{Specific frequency}

Numerous recent reviews have discussed the specific frequency $\left(S_{\mathrm{N}}\right)$ of GC systems in various galaxy types. The main discussion has centered on the significantly higher $S_{\mathrm{N}}$ values found in elliptical galaxies when compared with spiral galaxies (see for example, McLaughlin 1999; Ashman \& Zepf 1998). One goal of studying late-type galaxy GC systems is to establish what a "typical" spiral GC system looks like. If elliptical galaxies are built up from spiral-spiral mergers, then this is necessary to quantify predictions of the merger model. The specific frequency, $S_{\mathrm{N}}=N_{\mathrm{GC}} 10^{0.4\left(M_{V}+15\right)}$ (Harris \& van den Bergh 1981) gives the number of GCs, $N_{\mathrm{GC}}$, per unit galaxy luminosity, normalized to a galaxy with absolute $V$ magnitude of -15 . The characteristic specific frequency of GCs in spiral galaxies is not well established and this absence of firm constraints leads to uncertainties in detailed predictions for elliptical GC systems which follow from the merger model.

Using $M_{V}=-18.9$ for M 33 (Courteau \& van den Bergh 1999), and $N_{\mathrm{GC}}=75 \pm 14$ as found in the previous section, we find $S_{\mathrm{N}}=2.07 \pm 0.39$. An alternative approach in the literature attempts to account for variations in stellar mass-to-light ratios introduced because of different galaxy morphologies. Zepf \& Ashman (1993) normalized the number of GCs per unit stellar mass of the host galaxy,

$T \equiv \frac{N_{\mathrm{GC}}}{M_{\mathrm{G}} / 10^{9} M_{\odot}}$.

Conversion from galaxy luminosity to stellar mass is achieved by assuming a characteristic $M / L_{V}$ for each morphological type. While not an ideal procedure for individual galaxies, this method does tend to minimize differences in $S_{\mathrm{N}}$ generated by differences in stellar populations from one galaxy type to another. Assuming $M / L_{V}=5.0$ for Sc galaxies (Faber \& Gallagher 1979), the $T$ value for M 33 is $4.88 \pm 0.91$. The specific frequency found for seven other late-type spirals (type $\mathrm{Sb}$ or $\mathrm{Sc}$ ) has a mean $\langle T\rangle=1.2$ with dispersion, $\sigma=0.2$ (Kissler-Patig et al. 1999). Our estimates reveal that M 33 has the highest known $T$ value in any late-type spiral galaxy.

For comparison, $\langle T\rangle=3.0$ with a $\sigma=1.2$, (although the values are poorly defined for several of the galaxies), for four Sa and Sab galaxies (Kissler-Patig et al. 1999). Thus, Kissler-Patig et al. (1999) suggest that there may be an increase in the number of GCs per unit mass along the Hubble sequence of late-type galaxies, since early-type spirals have higher $T$ values. If the high specific frequency is supported in M 33, this may not be the case. In fact, the specific frequency values we derive for M 33 are within the range found for elliptical galaxies, although at the low end. If progenitor spirals in merging systems have specific frequencies as high as postulated in this work, then there would be less of a discrepancy in the number of GCs per unit mass between ellipticals and spirals.

\section{Summary}

We have searched HST archive WFPC2 images in the nearby spiral galaxy M 33 in order to extend our compilation of star clusters. Twenty eight fields have deep $V$ and $I$ band images, while an additional seven fields have $V$ filter images with at least $300 \mathrm{~s}$ exposures. Our previous survey using HST WFPC2 multiband images in $U, B$, and $V$ focussed on characterizing the young cluster population in M 33 (Papers I and II). The redder filter set used in this work complements and extends known cluster parameters in M 33, with deeper observations (completeness $m_{V} \sim 19.7$ ), and revealed intermediate age and old objects. 102 clusters were detected; 82 were previously unknown. Twenty-five clusters in our sample have $(V-I)$ colors typical of globular clusters (15 of these have not been previously detected), and eleven others have colors associated with intermediate age clusters (nine of these have not been previously detected). Future spectroscopic work is planned to obtain more precise ages, metallicities, as well as kinematic properties for these new objects. 
Luminosity functions for old $\left[(V-I)_{0}>0.78\right]$ M 33 clusters show a turnover at $M_{V} \sim-7.0$. The intermediate $\left[0.78 \geq(V-I)_{0} \geq 0.70\right]$ age object luminosity function does not show a turnover to a magnitude of $M_{V} \sim-6.6$.

Age estimates from comparison of measured cluster $(V-I)$ color with single stellar population models span a range from $<10^{7}$ years to $1510^{9}$ years. Because of the large uncertainties associated with age estimates based on one color, only the statistical distribution of ages is discussed. These were combined with theoretical $M / L_{V}$ ratios to estimate masses. We find that old M 33 clusters in our sample have masses from $10^{4} M_{\odot}-10^{6} M_{\odot}$, similar to the range found for Galactic globular clusters. We confirm the finding of Paper II that old clusters are more massive than young ones in this galaxy.

By combining all known globular cluster candidates from this study and the literature, we derive the surface density of GCs in M 33. The density of clusters in the inner regions of M 33, where surveys are more complete, are used to correct for incompleteness in the outer M 33 portions, resulting in an estimate of the total GC population in this galaxy of $75 \pm 14$. This gives a specific frequency, $S_{\mathrm{N}}$, of $2.07 \pm 0.39$, and $T$ of $4.88 \pm 0.91$. The actual frequency might be even higher, if cluster destruction rates are significantly lower in the outer M 33 regions, when compared with the inner regions. The numbers presented for M 33 are substantially higher than typical values in other late type spiral galaxies, but similar to values found for early-type spirals, and in the range found for elliptical galaxies.

Acknowledgements. We wish to thank an anonymous referee for useful comments and suggestions which improved this paper. Support for this work was provided by NASA grants NASA-GO08207 and AR-8001, from the Space Telescope Science Institute, which is operated by AURA, Inc. under NASA contract NAS5-26555.

\section{References}

Ashman, K., \& Zepf, S. 1998, Globular Cluster Systems (Cambridge: Cambridge University Press)

Bianchi, L., Chandar, R., \& Ford, H. C. 1999, Mem. SAIt, 70, 629

Bertelli, G., Bressan, A., Chiosi, C., Fagotto, F., \& Nasi, E. 1994, A\&AS, 106, 275

Chandar, R., Bianchi, L., \& Ford, H. C. 1999a, ApJS, 122, 431, Paper I

Chandar, R., Bianchi, L., \& Ford, H. C. 1999b, ApJ, 517, 668, Paper II

Chandar, R., Bianchi, L., Ford, H. C., \& Salasnich, B. 1999c, PASP, 111, 794, Paper III

Chandar, R., Bianchi, L., Ford, H. C., \& Sarajedini, A. 2001, in preparation

Chiosi, C., Vallenari, A., \& Bressan, A. 1997, A\&AS, 121, 301

Christian, C. A., \& Schommer, R. A. 1982, ApJS, 49, 405

Christian, C. A., \& Schommer, R. A. 1988, AJ, 95, 704

Courteau, S., \& van den Bergh, S. 1999, AJ, 118, 337

Dolphin, A. 2000, PASP, 112, 1397

Faber, S. M., \& Gallagher, J. S. 1979, ARA\&A, 32, 277

Freedman, W. L., Wilson, C. D., \& Madore, B. F. 1991, ApJ, 372,455

Harris, W. E., \& van den Bergh, S. 1981, AJ, 86, 1627

Hiltner, W. A. 1960, ApJ, 131, 163

Holtzman, J. A., Burrows, C. J., Casertano, S., et al. 1995, PASP, 107, 106

Kissler-Patig, M., Ashman, K., Zepf, S., \& Freeman, K. 1999, AJ, 118, 197

Kron, G. E., \& Mayall, N. U. 1960, AJ, 65, 581

McLaughlin, D. E. 1999, AJ, 117, 2398

Massey, P., Armandroff, T. E., Pyke, R., Patel, K., \& Wilson, C. D. 1995, AJ, 110, 2715

Melnick, J., \& D'Odorico, S. 1978, A\&AS, 34, 249

Mochejska, B. J., Kaluzny, J., Krockenberger, M., Sasselov, D. D., \& Stanek, K. Z. 1998, Acta Astron., 48, 455

Sarajedini, A., Geisler, D., Harding, P., \& Schommer, R. 1998, ApJL, 508, L37

Whitmore, B., Zhang, Q., Leitherer, C., et al. 1999, AJ, 118, 1551

Zepf, S., \& Ashman, K. 1993, MNRAS, 264, 611 